\title{
A NOTE ON A CHARACTERISTIC PROPERTY BASED ON ORDER STATISTICS
}

\author{
C. R. RAO AND D. N. SHANBHAG
}

(Communicated by Richard Durrett)

\begin{abstract}
It is shown that the extended version of the Puri-Rubin result given recently by Stadje (1994) is neither new nor the most general available in the literature.
\end{abstract}

Assuming $X_{1}$ and $X_{2}$ to be independent identically distributed random variables, Puri and Rubin [3] characterized, under some conditions, a certain class of distributions via the property that $\left|X_{1}-X_{2}\right| \stackrel{d}{=} X_{1}$. Recently, Stadje [9] proved, using Fourier analytic methods, that the Puri-Rubin result holds under a more general hypothesis. However, unfortunately the result of Stadje turns out to be neither new nor the most general available in the literature. We now make some observations in this respect.

Indeed, Lau and Rao [2] have pointed out in essence that the following theorem of theirs (i.e. Theorem 1) gives as a corollary the result that Stadje now proves. An elegant probabilistic proof based on exchangeability for the Lau-Rao theorem is given by Alzaid, Rao and Shanbhag [1]; see, also Ramachandran and Lau [5] for various other references on the theorem.

Theorem 1. Let $f$ be a non-negative real locally integrable Borel measurable function on $\mathbf{R}_{+}$, other than a function which is identically zero a.e. $[L]$, such that it satisfies

$$
f(x)=\int_{\mathbf{R}_{+}} f(x+y) d \mu(y) \quad \text { for a.a. } \quad[L] x \in \mathbf{R}_{+}
$$

for some $\sigma$-finite measure $\mu$ with $\mu(\{0\})<1$ (yielding trivially that $\mu\left(\{0\}^{c}\right)>0$ ), where $L$ corresponds to Lebesgue measure. Then, either $\mu$ is arithmetic with some span $\lambda$ and

$$
f(x+n \lambda)=f(x) b^{n}, \quad n=0,1, \ldots \quad \text { for a.a. } \quad[L] x \in \mathbf{R}_{+}
$$

with $b$ such that

$$
\sum_{n=0}^{\infty} b^{n} \mu(\{n \lambda\})=1
$$

Received by the editors August 18, 1994.

1991 Mathematics Subject Classification. Primary 62E10; Secondary 62 E05.

Key words and phrases. Integrated Cauchy function equation, Lau-Rao theorem, order statistics.

Research sponsored by the Army Research Office under Grant DAAHO4-93-G-0030.

(C)1996 American Mathematical Society 
or $\mu$ is non-arithmetic and

$$
f(x)=c \exp (-\eta x) \quad \text { for a.a. } \quad[L] x \in \mathbf{R}_{+}
$$

with $c$ as a constant and $\eta$ such that

$$
\int_{\mathbf{R}_{+}} \exp (-\eta x) d \mu(x)=1 .
$$

(Here 'a.e.' and 'a.a.' refer to 'almost everywhere' and 'almost all' respectively.)

Rossberg [8], Ramachandran [4], Rao [6] and Ramachandran and Lau [5] among others have produced versions of the following theorem. This theorem also follows as a corollary to Theorem 1; this is essentially an observation of Rao [6], and Ramachandran and Lau ([5; pages 44 and 45)] (see, also, Lau and Rao [2] for an observation in this connection). This latter result gives Stadje's result as an immediate corollary on taking $n=2$ and $i=1$, and ignoring the trivial case of $X_{i}=0$ a.s. The precise statement of the theorem is taken from Rao and Shanbhag [7]. (Incidentally, the statement as given in Ramachandran and Lau [5] is somewhat misleading as it involves an erroneous equation $\left(\begin{array}{l}n \\ r\end{array}\right) \int_{0}^{\infty} e^{-\lambda x} d F^{r}(x)=1$ when $F$ is exponential with parameter $\lambda$.) We have reproduced the proof of the theorem, based on Theorem 1 , for the benefit of the reader.

Theorem 2. Let $n \geq 2$ and $X_{1}, \ldots, X_{n}$ be i.i.d. random variables with d.f. $F$ that is not concentrated on $\{0\}$. Further, let $X_{1: n} \leq \cdots \leq X_{n: n}$ denote the corresponding order statistics. Then, for some $2 \leq i<n$,

$$
X_{i+1: n}-X_{i: n} \stackrel{d}{=} X_{1: n-i}
$$

where $X_{i: n-i}=\min \left\{X_{1}, \ldots, X_{n-i}\right\}$, if and only if one of the following two conditions holds:

(i) F is exponential.

(ii) $F$ is concentrated on some semilattice of the form $\{0, \lambda, 2 \lambda, \ldots\}$ with $F(0)=$ $\alpha$ and $F(j \lambda)-F((j-1) \lambda)=(1-\alpha)(1-\beta) \beta^{j-1}$ for $j=1,2, \ldots$ for some $\alpha \in$ $\left(0,\left(\begin{array}{c}n \\ i\end{array}\right)^{-1 / i}\right]$ and $\beta \in[0,1)$ such that $P\left\{X_{i+1: n}>X_{i: n}\right\}=(1-\alpha)^{n-i}$ (which holds with $\alpha=\left(\begin{array}{c}n \\ i\end{array}\right)^{-1 / i}$ or $\beta=0$ if and only if

$$
F(0)-F(0-)=\left(\begin{array}{c}
n \\
i
\end{array}\right)^{-1 / i}
$$

and

$$
F(\lambda)-F(\lambda-)=1-\left(\begin{array}{c}
n \\
i
\end{array}\right)^{-1 / i}
$$

for some $\lambda>0)$. (The existence of cases $\beta>0$ can easily be verified.)

Proof. We shall first establish the 'only if' part of the assertion. (1) implies that $P\left\{X_{1: n-i} \geq 0\right\}=(1-F(0-))^{n-i}=1$ and hence that $F$ is concentrated on $\mathbf{R}_{+}$. Hence, we can conclude from (1) that

$$
\left(\begin{array}{l}
n \\
i
\end{array}\right) \int_{\mathbf{R}_{+}}(\overline{\bar{F}}(x+y))^{n-i} F^{i}(d y)=(\overline{\bar{F}}(x))^{n-i}, \quad x \in \mathbf{R}_{+},
$$


where $\overline{\bar{F}}(x)=1-F(x), x \in \mathbf{R}_{+}$. As $P\left\{X_{1}=0\right\}<1$, we have then $\overline{\bar{F}}(0)>0$ and (2) implies that $\left(\begin{array}{c}n \\ i\end{array}\right)(F(0))^{i} \leq 1$ (where obviously we have used the property of $F$ that $F(0-)=0$ ). If $F$ is a non-arithmetic distribution (concentrated on $\mathbf{R}_{+}$) with $\left(\begin{array}{l}n \\ i\end{array}\right) F^{i}(0)<1$, then $(2)$ implies, in view of Theorem 1, that (i) holds. On the other hand, if $F$ is arithmetic with span $\lambda$ with $\left(\begin{array}{c}n \\ i\end{array}\right)(F(0))^{i}<1$, then the equation in question implies, in view of the cited theorem, that $F$ is such that $F(0)=\alpha$ and

$$
F(j \lambda)-F((j-1) \lambda)=(1-\alpha)(1-\beta) \beta^{j-1}
$$

for $j=1,2, \ldots$, for some $\alpha \in\left(0,\left(\begin{array}{l}n \\ i\end{array}\right)^{-1 / i}\right)$ and $\beta \in(0,1)$ such that

$$
P\left\{X_{i+1: n}>X_{i: n}\right\}=(1-\alpha)^{n-i} .
$$

Finally, if $\left(\begin{array}{c}n \\ i\end{array}\right)(F(0))^{i}=1$, then (2) is not met if $F$ is non-arithmetic, or arithmetic with some span $\lambda$ with at least two positive support points, but is met by any $F$ concentrated on $\{0, \lambda\}$ for some $\lambda(>0)$. This completes the proof of the 'only if' part of the assertion.

It is easily seen that (1) is equivalent to the condition that (2) holds with $\overline{\bar{F}}(0-)=$ 1. For all the distributions that we have arrived at in the proof of the 'only if' part, we have $\overline{\bar{F}}(0-)=1$ and (2) met. Hence, we have the 'if' part of the assertion, and consequently the theorem.

Remark 1. Suppose we consider a family of the distributions of the form in (ii), but not necessarily satisfying the condition that $P\left\{X_{i+1: n}>X_{i: n}\right\}=(1-\alpha)^{n-i}$. Then, if we take a fixed $\beta \in(0,1)$ and allow $\alpha$ to vary, we get for a sufficiently small $\alpha, P\left\{X_{i+1: n}>X_{i: n}\right\}<(1-\alpha)^{n-i}$, and for $\alpha=\left(\begin{array}{c}n \\ i\end{array}\right)^{-1 / i}$, we get $P\left\{X_{i+1: n}>X_{i: n}\right\}>$ $(1-\alpha)^{n-i}$; since we have now $P\left\{X_{i+1: n}>X_{i: n}\right\}$ to be a continuous function of $\alpha$, we have the existence of an $\alpha$ value such that $P\left\{X_{i+1: n}>X_{i: n}\right\}=(1-\alpha)^{n-i}$. This proves that the claim made by us under brackets immediately after the statement of the theorem is justified.

Remark 2. If $n=2$ and $i=1$, we get $\left(\begin{array}{c}n \\ i\end{array}\right)^{-1 / i}=1 / 2$. In this case, if neither $\alpha=1 / 2$ nor $\beta=0$, we get

$$
P\left\{X_{i+1: n}>X_{i: n}\right\}=1-P\left\{X_{1}=X_{2}\right\}=\{2(1-\alpha)(\alpha+\beta)\} /(1+\beta) ;
$$

consequently we have here $P\left\{X_{i+1: n}>X_{i: n}\right\}=(1-\alpha)^{n-i}$, i.e. the probability to be equal to $1-\alpha$, if and only if $\beta=1-2 \alpha$. One can hence see how Stadje's result follows as a corollary to Theorem 2 .

\section{REFERENCES}

1. A. Alzaid, C. R. Rao and D. N. Shanbhag, Solution of the integrated Cauchy functional equation using exchangeability, Sankhyā A 49 (1987), 189-194. MR 91e:62027

2. K. S. Lau and C. R. Rao, Integrated Cauchy functional equation and the characterization of the exponential law, Sankhyā 44 (1982), 72-90. MR 85j:62012a

3. P. S. Puri and H. Rubin, A characterization based on the absolute difference of two i.i.d. random variables, Ann. Math. Statist. 41 (1970), 251-255. MR 45:2836

4. B. Ramachandran, An integral equation in probability theory and its applications, Tech. Report, Indian Statistical Institute, 1980. 
5. B. Ramachandran and K. S. Lau, Functional equations in probability theory, Academic Press, New York, 1991. MR 93c:60016

6. C. R. Rao, An extension of Deny's theorem and its application to characterizations of probability distributions, A festschrift for Eric Lehmann, Wadsworth Statist./Probab. Ser., Wadsworth, Belmont, CA, 1983, pp. 348-366. MR 85d:62018

7. C. R. Rao and D. N. Shanbhag, Choquet-Deny type functional equations with applications to stochastic models, Wiley, New York, 1994.

8. H. J. Rossberg, Characterizations of the exponential and Pareto distributions by means of some properties of the distributions which the differences and quotients of order statistics are subject to", Math. Operationsforsch. Statist. 3 (1972), 207-216. MR 48:9915

9. W. Stadje, A characterization of the exponential distribution involving absolute differences of i.i.d. random variables, Proc. Amer. Math. Soc. 121 (1994), 237-243. MR 94g:62022

Center for Multivariate Analysis, 417 Classroom Building, Penn State University, University Park, Pennsylvania 16802

E-mail address: crr1@psuvm.psu.edu

Department of Probability and Statistics, University of Sheffield, Sheffield S37RH, UNITED KINGDOM 